\title{
Polarimetric measurements in prominences and "tornadoes" observed by THEMIS
}

\author{
Brigitte Schmieder $^{1}$, Arturo López Ariste ${ }^{2}$, Peter Levens ${ }^{1,3}$, \\ Nicolas Labrosse ${ }^{3}$ and Kévin Dalmasse D $^{1,4}$ \\ ${ }^{1}$ Observatoire de Paris, Meudon 92195, France \\ email: brigitte.schmieder@obspm.fr \\ ${ }^{2}$ IRAP, Toulouse, France, email: Arturo.LopezAriste@irap.omp.eu \\ ${ }^{3}$ University of Glasgow, Scotland, UK \\ ${ }^{4} \mathrm{CISL}$ and $\mathrm{HAO}, / \mathrm{NCAR}$ Boulder, USA
}

\begin{abstract}
Since 2013, coordinated campaigns with the THEMIS spectropolarimeter in Tenerife and other instruments (space based: Hinode/SOT, IRIS or ground based: Sac Peak, Meudon) are organized to observe prominences. THEMIS records spectropolarimetry at the He $\mathrm{I}_{3}$ and we use the PCA inversion technique to derive their field strength, inclination and azimuth.

All of the observed prominences are quiescent, as they were stable as filaments for at least three days and not eruptive. They present similar characteristics, they are highly dynamic and present horizontal magnetic fields. Statistically, the inclination from the local vertical is around 90 degrees, with some points around 60 and 120 degrees. The field strength is between 5 and 15 Gauss. We tested the effects of adding a turbulent field component to the horizontal field. For those pixels showing inclinations around 60 and 120 degrees, we find that such a model is compatible with the polarimetric observations. In some of these prominences, identified as "tornadoes" the field strength may reach 50 Gauss, and in the top of the tornadoes some points exhibit an inclination which cannot correspond to any model in our grid of models. We investigate different solutions.
\end{abstract}

Keywords. Solar prominences, magnetic field

\section{Introduction}

With the new armada of spacecraft observing the Sun (Hinode, SDO, IRIS) we are able to have an accurate view of the solar phenomena occurring in the corona. Movies obtained with high spatial and temporal resolution allowed us to discover the incredible dynamic nature of prominences, even the quiescent ones: up and down flows in quasi-vertical structures, rising bubbles and rotating - tornado-like - structures (Dudik et al. 2012; Orozco Suarez et al. 2012; Wedemeyer et al. 2013; Berger et al. 2014; Su et al. 2012). Spectroscopy is useful to analyze the real plasma motion and its physical conditions. Using $\mathrm{H} \alpha$ spectra obtained with the MSDP in the Meudon solar tower it was shown that, in prominences, the velocity vectors were not aligned with the apparent vertical structures, as the movies suggest, but have a significant angle with respect to the vertical (Schmieder et al. 2010). The tornado behavior was detected in the hot plasma $(\log \mathrm{T}>$ 6) surrounding the prominence legs by Hinode/EIS ( $\mathrm{Su}$ et al. 2014) but has not yet been confirmed in cooler plasma (Levens et al. 2015).

The highly dynamic plasma observed in prominences with the IRIS spectrograph could answer the question of short scale height of the plasma pressure compared with the common height of prominences (Schmieder et al. 2014). All the theoretical models are based on static structures (Aulanier \& Démoulin 1998; van Ballegooijen 2004; Dudik et al. 2008; Mackay et al. 2010). The plasma would be sustained in a pile of dips in sheared 


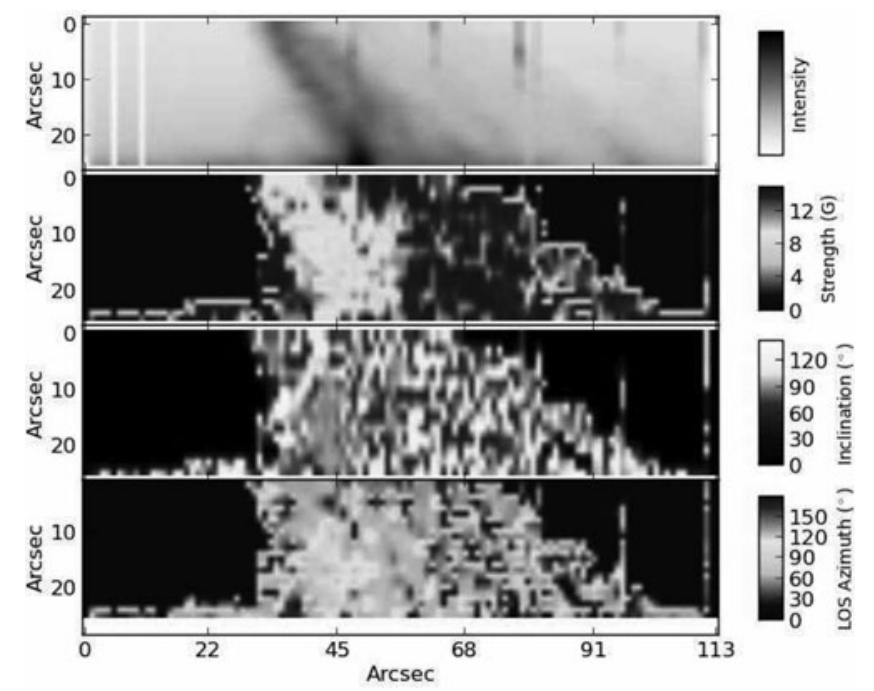

Figure 1. Magnetic field in the prominence on September 24, 2013: (from top to bottom) He I $\mathrm{D}_{3}$ intensity, field strength, local inclination, and line-of-sight azimuth (adapted from Schmieder et al. 2014).

arcades or in twisted flux ropes due to magnetic tension force or the presence of tangled magnetic field on small scales (van Ballegooijen \& Crammer 2010). This is consistent with previous measurements of the magnetic field in prominences. Polarimetry of prominences was achieved in the 1980s (Leroy et al. 1984) which showed that the prominence magnetic field vector was almost horizontal (60 to 90 degrees from the vertical). The inversion codes developed during this time period involved only the Hanle effect, neglecting the Zeeman effect (Bommier et al. 1994). It appears that the second effect cannot be neglected (Casini et al. 2003; López-Ariste and Aulanier 2007; Lites 2014). New inversion codes have been developed to include all those effects and are now being applied to new sets of full Stokes vector observations.

In this paper we present some magnetic field maps of prominences observed during two campaigns involving the French telescope THEMIS in the Canary Islands with its MTR mode.

\section{Observations}

During two international campaigns devoted to the observations of prominences in 2013 and 2014, the THEMIS/MTR instrument, located in the Canary Islands (LópezAriste et al. 2000) measured spectropolarimetry at the $\mathrm{He} \mathrm{I} \mathrm{D}_{3}$ line in prominences. The spectrograph slit was oriented parallel to the local limb. The double-beam polarimetry required the use of a grid mask that presented us with three segments 15.5 arcsec wide along the slit, but masked regions of 17 arcsec between each segment. The masked regions allowed us to obtain a double image with opposite polarization, but it also meant that in order to get continuous coverage of the prominence along the slit, we also had to scan in this direction by one step of 15 arcsec. This mode of scanning along the slit introduced the jumps and dark lines in the images that are presented in Figures 1 and 2.

In addition to that scan along the slit, a scan perpendicular to the slit with steps of 2 arcsec from the limb to the top of the prominence was achieved. Fields of view 


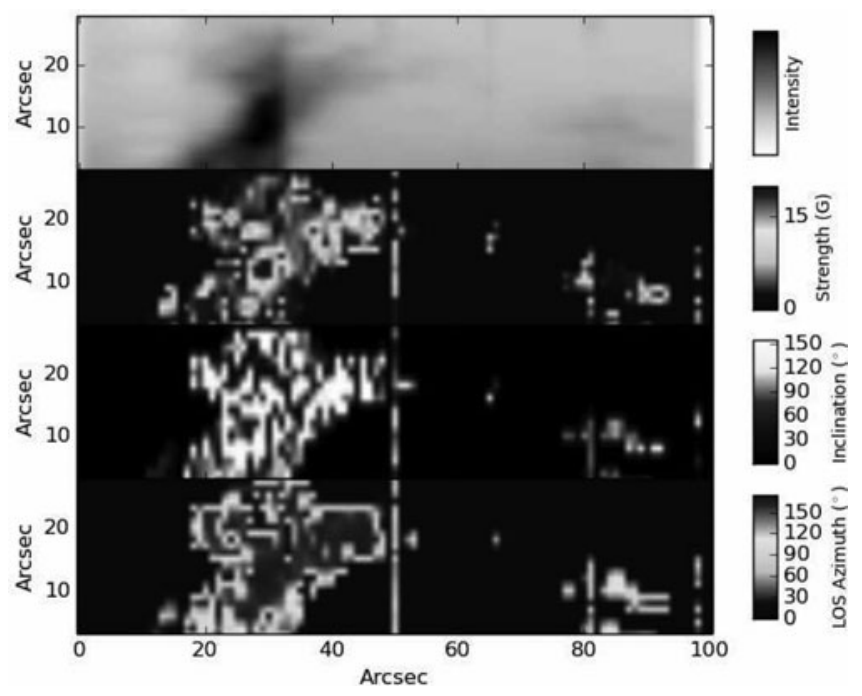

Figure 2. Magnetic field in a tornado observed on May 23, 2014: (from top to bottom) He I $\mathrm{D}_{3}$ intensity, field strength with some saturated points, local inclination, and line-of-sight azimuth.

of 120 arcsec $\times 20-40$ arcsec were covered in about half an hour to one hour with an exposure time of 2 seconds per Stokes parameter and scan position. Full polarimetry with beam-exchange was done with a modulation cycle of 6 images, spanning the three Stokes parameters with either positive or negative sign measured in every beam, and the simultaneous double beam measuring the opposite sign. Each Stokes parameter is thus measured in the same camera pixel at two different times and in two different pixels at the same time. This symmetry of measurements results in high polarimetry precision and a reduction in the systematic errors to a fourth order perturbation of the signal. Each cycle was repeated five times to increase $\mathrm{S} / \mathrm{N}$ ratios.

\section{Data reduction}

The raw data of the THEMIS/MTR mode was reduced with the DeepStokes procedure (López Ariste et al. 2009). Data reduction included flat-fielding, dark current and bias subtraction, wavelength calibration and, particularly, a careful handling of the polarization signals. The results of the data reduction are cubes of spectra for the He $\mathrm{I}_{3}$ line in intensity, linear polarization (both $Q$ and $U$ ) and circular polarization, for all points along the slit and all positions of the double scan. $\mathrm{S} / \mathrm{N}$ ratios are better than $10^{3}$ at the core of the He $\mathrm{I} \mathrm{D}_{3}$ line in the central parts of the prominence for all three Stokes parameters.

The Stokes profiles are the inputs to an inversion code based on Principal Component Analysis (PCA) (López-Ariste \& Casini 2002; Casini et al. 2003) that efficiently compares the observed profile against those generated with known models of the polarization profiles of $\mathrm{He} \mathrm{I} \mathrm{D}_{3}$ and stored in a database (see López-Ariste \& Casini 2002 for more details). The comparison is made independently pixel by pixel. The database we used contains about 90000 profiles computed as the emission of a single He atom in its triplet state modeled with the 5 levels of lower energy of the He triplet system. The atom is polarized by the anisotropic radiation of the photosphere below the prominence. The 


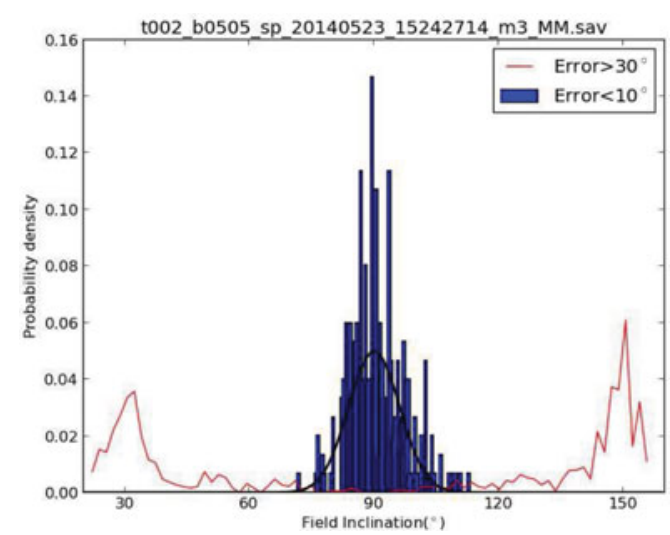

Figure 3. Histogram of the inclination of the magnetic field in the tornado. Solid bars are used for accurate inversions, while the thin line is used for erroneous inversions. These last ones are due to magnetic topologies not present in our database.

atomic polarization depends on the height of the atomic system and is one of the free parameters of the model. Collisions are not taken into account. The atomic polarization of the He atom is modified by a single vector magnetic field with strength, inclination and azimuth. The Hamiltonian of the atom includes all terms with its Zeeman sublevels splitting linearly with the magnetic field. We solve the density matrix of the atom in statistical equilibrium; the solution contains all populations and quantum coherences, including atomic alignment and orientation, for all the levels involved in the He triplet atom model. The Hanle effect of every level is thus computed as well as the Zeeman effect. From the resulting populations and coherences we compute the polarization dependent emission terms, in whatever direction we are observing. The scattering angle is thus a free parameter of the model too. Several million profiles are computed and used to build the database, keeping just those which are different enough and rejecting others so that the database fills the space of possible profiles as homogeneously as possible, while keeping the size small.

The He I D $587.6 \mathrm{~nm}$ line appears as a doublet. The two spectroscopic components have a different sensitivity to the Hanle effect and are far from being saturated in prominence conditions. This helps constraining ambiguities and error bars. This is in contrast with the other important He line in prominences, the He $1083 \mathrm{~nm}$ line. The analysis of the information content of the two He lines made by Casini et al. (2003), López Ariste and Casini (2005) and Casini et al. (2009) using PCA-based inversion codes demonstrated that the He $\mathrm{I}_{3}$ line better constraints the solution, with better determined field strengths and inclinations that seldom suffer from the 90 degrees ambiguity. Further, the PCA inversion code provides a solution and error bars for every pixel in the observation. This error bar is computed as the standard deviation over all solutions in the database that fit the observation within predefined margins. They include noise-related errors, but also point to ambiguities. Well inverted profiles can be declared free of ambiguities other than the ubiquitous 180 degree ambiguity in the azimuth of the field (in the observers's reference frame). Because of this, the few situations in which the 90 degrees ambiguity could appear in our solutions, would be finger-pointed by large error bars of 90 degrees and more. We have never found in this dataset problems of this kind due to the 90 degree 
ambiguity, unlike the case of the polarimetry analysis of a prominence observed with the He 1083nim line (Orozco Suarez et al. 2014).

After comparison of any observed profile with those in the database, the most similar is kept as the solution. The main output is the vector magnetic field and we check that the free parameters of the model, height above the photosphere, and scattering angle are in a typical range where the polarization of $\mathrm{D}_{3}$ is not so dependent on their values and consistent with the observations. Error bars are determined for those parameters as well by doing some statistics on all other models which are sufficiently similar to the observed ones, though not as similar as the one selected as the solution. It is important to stress that although there is always one case in the database that is the most similar to the observed one, this does not mean that it is a good fit to all of the observed profiles. It is thus important to keep a measure of how similar they are and also to check that all conclusions about the magnetic field strength or orientation are based upon sets of profiles that correctly represent the observation.

\section{Results}

During the 2013 THEMIS campaign more than 10 prominences were observed. One of them has been intensively studied and the results concerning the polarimetry and also its dynamic behavior, principally observed in spectroscopy using the IRIS spectrograph, have been published in Schmieder et al. (2014).

The THEMIS campaign in 2014 was performed during 10 days in May and 10 days in July. More than 150 scans of different prominences were made. Some of them can be identified as tornadoes in the context observations. In fact, the AIA movies in $171 \AA$ revealed an apparent rotation of the legs of the prominences. For some of these observations EIS Dopplershift maps indicated a characteristic pattern with red and blueshift in each side along the tornado axis. The polarimetry of the prominences has been achieved for all these prominences (López Ariste 2015, this issue) and the PCA inversion code was applied to it. In many points only one clear solution was proposed and it is generally a horizontal magnetic field. The error bars are, in these cases, small. The overwhelming majority of non-horizontal fields found by the inversion code have large error bars $(\sim 30$ degrees) but not enough to fall with a vertical field solution. There are places where the simple model of one magnetic field per pixel fails.

Figure 1 presents the maps of the September 24, 2013 prominence obtained after inversion of the Stokes parameters recorded in the He $\mathrm{I} \mathrm{D}_{3}$ line with THEMIS/MTR: (a) Intensity, (b) Magnetic field strength (c) Inclination, (d) line-of-sight Azimuth. The magnetic field strength is lower than 15 Gauss. The angle origin of inclination is the local vertical, the origin of the azimuth is a plane containing the line of sight and the local vertical. The inclination values indicate mainly an horizontal field vector. The other values are gathered around 60 and 120 degrees. These non-horizontal fields show large error bars which, as we said, are indication of a wrong model for the magnetic field. In this particular case it was shown that these pixels with inclinations around 60 and 120 degrees could be explained with two magnetic field components per pixel: one being horizontal and the other turbulent (Schmieder et al. 2014).

Figure 2 presents the maps of the May 23, 2014 tornado: We note that the magnetic field strength reaches 50 Gauss in a few points in the tornado (saturated in the image), much larger value than in the main body of prominences. We have computed the histogram of the inclination for all the points in the tornado (Figure 3). The main peak is centered on 90 degrees, indicating that the magnetic field is horizontal. Those results have small error bars and we are confident that the horizontal magnetic field of the 
prominence is correctly measured. The two secondary peaks at inclinations of 30 degrees and 150 degrees, on the other hand, present large error bars ( 30 degrees). Once again we conclude from this that the magnetic field model of one field per pixel used by the PCA inversion code is not valid. We can definitely exclude a vertical field as solution, which would have been correctly inverted. Several possibilities mixing several magnetic components as in the case of the prominence in Fig. 1 are being explored.

\section{Conclusion}

The tornadoes and prominences have the same magnetic structure with a main dominant horizontal field component. It confirms that these tornado-like structures $(\mathrm{H} \sim 20$ $\mathrm{Mm}$ ) are the legs (barbs or footpoints) of prominences as was suggested by Wedemeyer et al. (2013) and may differ from the small scale magnetic tornado initiated over photosphere vortex in the chromosphere (H>2 Mm) (Wedemeyer \& Steiner 2014).

\section{Acknowledgements}

We would like to thank the team of THEMIS for aquiring the observations. The National Center for Atmospheric Research is sponsored by the National Science Fondation.

\section{References}

Aulanier, G. \& Démoulin, P. 1998, ApJ 703, 114

Berger, T. 2014, in: B. Schmieder, J. M. Malherbe \& S. T. Wu (eds.), IAU Symposium No. 300 (Cambridge: CUP), p. 15

Bommier, V., Landi Degl'Innocenti, E., Leroy, J.-L., \& Sahal-Bréchot, S. 1994, Solar Phys. 154, 231

Casini, R., López Ariste, A., Tomczyk, S., \& Lites, B. W. 2003, ApJ (Letters) 598, L67

Casini, R., Bevilacqua, R., \& López Ariste, A. 2005, ApJ 622, 1265

Casini, R., López Ariste, A., Paletou, F., \& Léger, L. 2009, ApJ 703, 114

Dudík, J., Aulanier, G., Schmieder, B. et al. 2008, Solar Phys. 248, 29

Dudík, J., Aulanier, G. Schmieder, B., Zapiór, M. \& Heinzel, P. 2012 ApJ 761, 9

Labrosse, N., Heinzel, P., Vial, J.-C., Kucera, T., Parenti, S., Gunár, S., Schmieder, B., \& Kilper, G. 2014, Space Science Review 151, 243

Leroy, J. L., Bommier, V., \& Sahal-Bréchot, S., 1984, Solar Phys. 83, 135

Levens, P., Labrosse, N., Fletcher, L., \& Schmieder, B. 2015, A\& A submitted

Lites, B. 2014, in: B. Schmieder, J. M. Malherbe \& S. T. Wu (eds.), IAU Symposium 300 (Cambridge: CUP), p. 101

López Ariste, A., Rayrole, J., \& Semel, M. 2000, A\&AS 142, 137

López Ariste, A. \& Casini, R. 2002, ApJ 575, 529

López Ariste, A. \& Casini, R. 2005, A\&A 436, 325

López Ariste, A. \& Aulanier, G. 2007, in: P. Heinzel, I. Durotovič, R.Rutten (eds.), The Physics of Chromospheric Plasmas, ASP Conf. Series 368 (San Francisco: ASP), p. 291

López Ariste, A., Asensio Ramos, A., Manso Sainz, et al. 2009, A\& A 501, 729

Mackay, D. H., Karpen, J. T., Ballester, J. L., Schmieder, B., \& Aulanier, G. 2010, Space Science Review 151, 333

Orozco Suarez, D., Asensio Ramos, A. \& Trujillo Bueno, J. 2012 ApJ (Letters) 761, L25

Orozco Suarez, D., Asensio Ramos, A. \& Trujillo Bueno, J. 2014 A\&A 566, A46

Schmieder, B., Chandra, R., Berlicki, A., \& Mein, P. 2010, A $E A$ 514, A68

Schmieder, B., Tian, H., Kucera, T. et al. 2014, A\&A 569, A85

Schmieder, B., Kucera, T., Knizhnik, K. et al. 2013, A\&A 28, 490

Su, Y., Gomory, O., Veronig, A. et al. 2014, ApJ (Letters) 785, L2

Su, Y. \& Wang, T., V 2012, ApJ (Letters) 756, L41

van Ballegooijen, A. A. 2004, ApJ 612, 519 
van Ballegooijen, A. A. \& Crammer, S. R. 2010, ApJ 711, 164

Wedemeyer, S., Scullion, E., Rouppe von der Voort, L. et al. 2013, ApJ 774, 123

Wedemeyer, S. \& Steiner, O. 2014, PASJ 66 (SP1), S10 (1-8) 